\title{
Basic fibroblast growth factor induces miR-134 upregulation in astrocyte for cell
}

maturation

Tadahiro Numakawa ${ }^{1, * \#}$, Shingo Nakajima ${ }^{1 \#}$, Noriko Yamamoto ${ }^{1}$, Yoshiko Ooshima ${ }^{1}$, Haruki Odaka $^{1,2}$, Kazuo Hashido ${ }^{3}$, Naoki Adachi ${ }^{1}$, Hiroshi Kunugi ${ }^{1}$

1 Department of Mental Disorder Research, National Institute of Neuroscience, National Center of Neurology and Psychiatry (NCNP), Tokyo, Japan

2 Department of Life Science and Medical Bioscience, School of Advanced Science and Engineering, Waseda University, Tokyo, Japan

3 Administrative Section of Radiation Protection, National Institute of Neuroscience, NCNP, Tokyo, Japan

*Tadahiro Numakawa, Ph.D. (corresponding author)

Department of Mental Disorder Research, National Institute of Neuroscience, National Center of Neurology and Psychiatry, 4-1-1, Ogawa-Higashi, Kodaira, Tokyo, 187-8502, Japan Tel: +81-42-341-2711 ext. (5132), Fax: +81-42-346-1744, E-mail: numakawa@ncnp.go.jp

\# T.N. and S.N. contributed equally to the present study. 


\begin{abstract}
Evidence suggests that neuronal microRNAs (miRs) contribute to synaptic plasticity, although a role of glial miRs have been unknown. Growth factors including brain-derived neurotrophic factor (BDNF) regulate neuronal functions via upregulation of miRs, while possible influences on expression/function of glial miRs have not been fully understood. Here, we report that basic fibroblast growth factor (bFGF) increased miR-134 expression in astrocyte. The miR-134 was upregulated through stimulating extracellular signal-regulated kinase and phosphatidylinositol 3-kinase signaling, because inhibitors for each signaling blocked the miR-134 induction by bFGF. We also found upregulation of glial fibrillary acidic protein (astrocyte marker) and decreased extracellular concentration of glutamate after miR-134 overexpression and bFGF application, suggesting that astroglial cell maturation is enhanced by bFGF through induction of miR-134.
\end{abstract}

Keywords; bFGF, BDNF, microRNA, miR-134, glutamate, astrocyte 


\section{Introduction}

MicroRNAs (miRs), a highly conserved noncoding small RNAs, have been recognized to be a regulator for various physiological aspects including neural development via inhibiting the function of mRNA of target genes [1]. Both negative (e.g. miR-34a, -134, -137, and -219) and positive (e.g. miR-124, -132, and -188) functions of neural miRs in the neuronal maturation and synaptic plasticity have been demonstrated [2]. Recently, it has been suggested that the dysregulation of miR-132 is involve in the abnormality of neurodevelopment and neuro-morphology in schizophrenia [3]. In addition, interfering of miR-134 function in mice by antagomir leads to reduction of evoked seizures and neuroprotection [4], suggesting a possible therapeutic role of miRs in brain diseases.

Studies have demonstrated that the miR expression is regulated by growth factors in neurons [5-7]. For example, BDNF/TrkB (brain-derived neurotrophic factor and its high affinity receptor) system upregulates expression levels of miR-212/132 through activating MSK (mitogen- and stress-activated kinase) 1 and CREB (cAMP-response element binding protein) pathways [5]. Glial cell line-derived neurotrophic factor (GDNF) also regulates miRs (e.g. miR-188-5P, miR-434-3P, and miR-340-P) expression, and protects dopaminergic cell line against cell death induction caused by 6-hydroxydopamine [7]. Previously, we showed that basic fibroblast growth factor (bFGF) enhanced miR-132 expression not only in cortical neurons but also in astrocytes, while BDNF-dependent miR-132 induction was observed in only neurons and plays a role for synaptic protein expression [8]. On the other hand, miR-125b [9] and miR-155 [10] have been identified as astroglial miRs. However, function of glial miRs induced by growth factors has been poorly understood. Especially, though bFGF is a well-known regulator for cell differentiation and survival in neurons [11], glial miR induced by bFGF and its physiological role has not been clarified.

MiR-134, one of brain-specific miRs, localizes in dendrites, and regulates dendritic spine size via suppressing levels of Limk1 (Lim-domain-containing protein kinase 1) mRNA in hippocampal neurons [12]. MiR-134 has a role in neuronal development such as proliferation of neural precursor cell and embryonic neuronal maturation [13]. Increased miR-134 is important for the dendritic maturation induced by BDNF through regulating myocyte enhancing factor 2 (Mef2), a transcription factor [14]. These reports show functions of neuronal miR-134 and its interaction with growth factors including BDNF.

In the present study, we found that significant miR-134 upregulation after bFGF stimulation in astrocytes, and that overexpression of miR-134 increased expression levels of astroglial marker proteins and glutamate transporter, suggesting a novel role of miR-134 in astroglial maturation and glial function. 


\section{Materials and Methods}

Antibodies and materials

Anti-phosphorylated or total extracellular signal-regulated kinase1/2 (ERK1/2), and

anti-phosphorylated or total Akt antibodies were purchased from cell signaling technology Inc.

(Danvers, MA, USA). U0126 and LY294002 were also from the cell signaling technology Inc.

Both anti-S-100 and anti-aldehyde dehydrogenase 1 family, member L1 (ALDH1L1) antibodies were obtained from Abcam plc (Cambridge, UK). Anti-glial glutamate transporter GLT-1

(EAAT2) antibody was purchased from Santa Cruz Biotechnology, Inc. (Dallas, TX, USA).

Anti- glial fibrillary acidic protein (GFAP) and anti- $\beta$-actin antibodies were obtained from Chemicon International, Inc. (Temecula, CA, USA) and Sigma-Aldrich (St. Louis, MO, USA), respectively. Taqman ${ }^{\circledR}$ microRNA assays (miR-9: 000583, miR-124a: 001182, miR-132: 000457, miR-134: 001186, and miR-16: 000391), Taqman ${ }^{\circledR}$ microRNA reverse transcription kit, epidermal growth factor (EGF), Alexa Fluor ${ }^{\circledR} 488$ goat anti-rabbit IgG, Hoechst 33342, Dulbeco's modified Eagle medium: Nutrient Mixture F-12 (DMEM/F12), and minimum essential medium (MEM) from Life technologies corporation (Carlsbad, CA, USA) were used in this study. Takeda Pharmaceutical company Ltd., and Sumitomo Co. Ltd. kindly donated BDNF. bFGF was purchased from PeproTech (Rocky Hill, NJ, USA). Insulin-like growth factor -1 (IGF-1) and GDNF were purchased from R\&D systems (Minneapolis, MN, USA) and Wako Pure Chemical Industries Ltd. (Osaka, Japan), respectively.

\section{Cell cultures}

Primary cortical neurons and astrocytes were prepared from cerebral cortex of wistar rats at postnatal day 1-2 as described previously [8]. Neural cortical cells were cultured with DMEM/F12 containing heated-inactivated 5\% horse serum and 5\% fetal bovine serum (FBS), and $2 \mu \mathrm{M}$ cytarabine to prevent glial cell survival. At 4-5 days in vitro (DIV), the neuronal cultures were treated with $100 \mathrm{ng} / \mathrm{ml} \mathrm{BDNF}$ or bFGF for 24 hours. Astrocyte pure cultures were maintained with MEM-based growth medium supplemented with $100 \mu \mathrm{g} / \mathrm{l} \mathrm{EGF,} 20 \mathrm{mM}$ glucose, $25 \mathrm{mM} \mathrm{NaHCO}_{3}, 5 \% \mathrm{FBS}$, and $0.5 \mathrm{mM}$ glutamine. When the confluency of the astrocytes was reached to $70-80 \%$, culture medium was replaced with fresh one without EGF for 48 hours before stimulation by growth factors. The pre-miR-134 (CAGGGUGUGUGACUGGUUGACCAGAGGGGCGUGCACUUUGUUCACCCUGUGGG CCACCUAGUCACCAACCCUC) with 5'- and 3'- franking region was inserted to BamH I/Hind III site of pBApo-CMV Neo vector (TAKARA BIO Inc., Shiga, Japan). Transfection of 3 $\mu \mathrm{g}$ of the miR-134 expression vector into astrocytes was conducted with Lipofectamine 2000 (Life Technologies) according to manufacturer's protocol. After additional 48 hour maintenance, transfected cells were used for experiments. We confirmed that the transfection efficiency was 
approximately $77.4 \pm 8.7 \%$ (the ratio: the number of GFP-positive cells to the number of total nuclear stained with Hoechst 33342) using GFP construct (pAcGFP1-N1; Clontech laboratories, Inc., Mountain View, CA, USA),

The measurement of miRs expression

Extraction of total RNA including miRs from cultures was performed with mirVana ${ }^{\mathrm{TM}}$ miRNA isolation kits (Ambion, Austin, TX, USA) according to manufacturer's instruction. The concentration of total RNA was measured (as optical density, OD, at $260 \mathrm{~nm}$ ) by using ND-1000 spectrophotometer (Nanodrop, Wilmington, DE, USA), and quality of RNA was determined with the ratio value of OD260/OD280 (> 1.8). Then, $100 \mathrm{ng}$ of total RNA were applied to reverse-transcription with Taqman ${ }^{\circledR}$ primer and Taqman ${ }^{\circledR}$ microRNA reverse transcription kit. The quantification of miR concentration in extracted samples was carried out by real-time PCR using specific Taqman ${ }^{\circledR}$ probe with 7900 HT Fast Real-Time PCR System (Life Technologies Corporation). The data was analyzed by SDS 2.2 real-time PCR data analysis software (Life Technologies Corporation). In this study, the each concentration value was normalized by levels of miR-16 in the same sample.

\section{Western blotting}

Total lysate from astroglial cultures were collected with lysis buffer which composed of $1 \%$ SDS, $10 \mathrm{mM} \mathrm{Na}_{4} \mathrm{P}_{2} \mathrm{O}_{7}, 10 \mathrm{mM}$ Tris- $\mathrm{HCl}, 10 \mathrm{mM} \mathrm{NaF}, 5 \mathrm{mM}$ EDTA, $2 \mathrm{mM} \mathrm{NaVO}_{4}$ and $1 \mathrm{mM}$ PMSF. The protein concentration in each sample was measured with Pierce ${ }^{\circledR}$ BCA protein assay (Thermo scientific, IL, USA), before equivalent amounts of total proteins were applied to each electrophoresis. Separated proteins on acryl-amide gel were blotted to PVDF membrane (Millipore, MA, USA), then, incubation with primary antibody after blocking with 5\% skim milk diluted in TBS $\{0.1 \mathrm{M}$ Tris- $\mathrm{HCl}$ (pH 7.4) in saline $\}$ was performed. The mouse- (Jackson ImmunoResearch Europe Ltd., Suffolk, UK) or rabbit- (Rockland Immunochemicals, Inc., Gilbertsville, PA, USA) secondary antibody was used after several times of washing with TBS. The immunoreactivity visualized by Immunostar ${ }^{\circledR}$ reagents (Wako Pure Chemical Industries Ltd., Osaka, Japan) and ECL films (GE healthcare UK Ltd., Buckinghamshire, UK) was obtained. The optical density of each protein expression was determined by CS Analyzer Version 3.00.1011 (ATTO Co., Tokyo, Japan).

\section{Cell survival}

MTT \{3-(4,5-dimethylthiazol-2-yl)-2,5-diphenyltetrazolium bromide, yellow tetorazolium salt\} assay was performed as previously reported [15]. Briefly, cultured medium was exchanged to fresh DMEM/F12 containing $0.5 \mathrm{mg} / \mathrm{ml}$ MTT. After $1.5 \mathrm{~h}$ incubation with the MTT solution, 
cell viability which can be recognized as deep blue formazan product from the yellow tetorazolium salts was measured at OD570 with iMark micro plate reader (Bio-Rad Laboratories, Hercules, CA, USA).

\section{Immunohistochemistry}

Cultured astrocytes were fixed with $4 \%$ paraformaldehyde in phosphate buffered saline (PBS)

for $20 \mathrm{~min}$. After several time washing with PBS, incubation with anti-GFAP antibody (1:1000) with $0.2 \%$ Triton-X (sigma) and 10\% FBS in PBS was performed over-night followed by washing with fresh PBS. After incubation with secondary antibody, immunofluorescence image was captured with a fluorescent microscope (Observer Z1, Carl Zeiss, Oberkochen, Germay). The value of fluorescence intensity from 32-37 cells selected randomly in 4 dishes for each experimental condition was calculated.

\section{The measurement of glutamate release}

The amount of extracellular glutamate in cultured astroglial cells was measured according to the previous study [15]. Briefly, treatment with bFGF (24 hours) or overexpression of miR-134 (48 hours after transfection) was performed before washing 4 times with the modified Hepes-buffered Krebs Ringer solution (KRH; containing $130 \mathrm{mM} \mathrm{NaCl}, 10 \mathrm{mM}$ glucose, $5 \mathrm{mM}$ $\mathrm{KCl}, 1.8 \mathrm{mM} \mathrm{CaCl}_{2}, 1.2 \mathrm{mM} \mathrm{NaH}_{2} \mathrm{PO}_{4}, 1 \% \mathrm{BSA}$, and $25 \mathrm{mM}$ Hepes, $\mathrm{pH}$ 7.4). Then, the cultured astroglial cells were incubated with fresh KRH buffer for $20 \mathrm{~min}$. Afterward, collected KRH samples were applied to the high-performance liquid chromatography (HPLC; Shimazu Co., Kyoto, Japan).

\section{Statistical analysis}

Data were presented as mean \pm standard deviation (SD). Statistical analysis between two groups was performed with student's $t$-test. The data among multiple groups were analyzed by one- or two-way analysis of variance (ANOVA) with Bonferroni's multiple comparison post-hoc test (SPSS Japan, Tokyo, Japan). Probability values less than 0.05 was defined as statistically significant. 


\section{Results}

bFGF increased miR-134 expression in astrocytes.

As we previously reported [8], miR-132, (but not miR-9, or -124a), was increased by BDNF and bFGF application in cultured cortical neurons (Fig. 1A). bFGF induced the expression of neural miR-134 although the increment was lower than miR-132 increase (Fig. 1A). Because it has been reported that astrocytes also express bFGF receptor [16], we tested whether bFGF increases brain-specific miRs in astrocyte. Though the expression levels of miR-132 were significantly increased by bFGF in astrocytes, an induction of miR-134 was much higher compared with that of miR-132 (Fig. 1B). To confirm the positive effect of bFGF on glial miR-134 expression, a dose-dependency of the expression was examined. Glial miR-134 expression was dose-dependently enhanced by bFGF (Fig. 1C). Previously, we confirmed significant activation of ERK, one of the intracellular signaling molecules, after bFGF, IGF-1, or EGF addition to astrocytes, suggesting an existence of specific receptor for each factor [8]. Interestingly, bFGF induced marked upregulation of miR-134 in astroglial cultures, while the other growth factors (including BDNF, IGF-1, GDNF, and EGF) did not increase miR-134 levels (Fig. 1D).

The involvement of ERK and PI3K signaling in the bFGF-induced miR-134.

bFGF activates ERK and phosphatidylinositol 3-kinase (PI3K) signaling in astrocytes [17]. To clarify the contribution of intracellular signaling to the bFGF-increased miR-134, astrocytes were exposed to bFGF in the presence of MEK (MAP kinase kinase, upstream of ERK) inhibitor, U0126. U0126 completely inhibited both ERK activation and miR-134 upregulation induced by bFGF (Fig. 2A and 2B). LY294002, PI3K inhibitor, also inhibited Akt activation and significantly diminished the miR-134 induction by bFGF (Fig. 2C and 2D). These results suggest that ERK and Akt signaling are involved in the miR-134 induction by bFGF.

\section{Overexpression of miR-134 did not affect cell survival in astrocytes.}

To investigate physiological role of glial miR-134, we performed miR-134 overexpression. Transfection of the miR-134 construct drastically increased miR-134 expression (Fig. 3A). Because bFGF plays a role in cell proliferation and differentiation in astrocytes [18, 19], we checked cell viability after exogenous miR-134 induction, and found that this increment of miR-134 had no effect on cell viability (Fig. 3B). The basal levels of pERK and pAkt were not changed by the increase of miR-134 (Fig. 3C).

miR-134 overexpression enhances glial maturation.

Next, when we measured an immunoreactivity of glia fibrillary acidic protein (GFAP, astrocytic 
cytoskeleton protein), increased intensity of immunofluorescence by the miR-134

overexpression was observed (Fig.4A). In addition to increased GFAP, upregulation of other glial specific proteins including ALDH1L1 and S-100 expression after the miR-134

overexpression in astroglial cultures was observed (Fig. 4B). As expected, bFGF also increased the expression levels of astroglial markers, GFAP and ALDH1L1 (Fig. 4C).

To further assess physiological role of glial miR-134, we also examined the glial glutamate transporter, GLT-1, in the miR-134 overexpressed astroglial cultures, because the regulation of transmitter concentration through specific transporters is a main function of glial cells to maintain neurotransmission [20]. Importantly, expression levels of GLT-1 were increased by the overexpression of miR-134 and treatment of bFGF (Fig. 4D, 4E). Furthermore, both miR-134 overexpression and bFGF treatment decreased the extracellular glutamate concentration (Fig. $4 \mathrm{~F})$. 


\section{Discussion}

Here, we found that bFGF increased miR-134 expression in astrocytes. The upregulation of miR-134 was inhibited by co-application with an inhibitor for ERK- or Akt-signaling, respectively. Overexpression of miR-134 caused an increase in the expression of glial specific proteins and glutamate transporter, and decreased extracellular glutamate concentration.

Evidence suggests important roles of miR-134 in neurons. Expression of neural miR-134 is linked to cell maturation and localized in dendrites to regulate hippocampal spine size via inhibiting Limk1 mRNA expression [12]. The translational repressor Pum-2 (Pumilio2) is another target of miR-134, which is involved in the dendritic outgrowth mediated by Mef- 2 in an activity-dependent manner [14]. In this study, we found a glial role of miR-134 in relation to bFGF function in astrocyte. It is well known that bFGF regulates astroglial development including cell differentiation and proliferation [18, 21]. Remarkably, FGF-2/FGF-5 double mutant mice exhibited significantly reduced astroglial marker proteins such as GFAP and S-100 in the prefrontal cortex [21]. Astroglial proliferation stimulated by bFGF was also reported [18]. In our pure astroglial cultures, overexpression of miR-134 and treatment with bFGF clearly increased not only the expression of glial specific proteins (GFAP, ALDH1L1 and S-100) but also the expression of GLT-1 without any effect on cell viability, suggesting that bFGF-increased miR-134 contributes to maturation of astrocytes.

In both neuronal and astroglial cultures, we recently found an induction of miR-132 by bFGF through activating ERK pathway [8]. The ERK signal cascade is thought to have pivotal roles in the synaptic plasticity $[22,23]$. Importantly, ERK signaling stimulated by bFGF is involved in the process extension of astrocytes [17]. In the present study, it was revealed that miR-134 worked downstream of ERK activation in astrocytes and that the function of miR-134 is possibly cell maturation, since miR-134 overexpression resulted in increased levels of glial proteins and GLT-1.

The increase of hippocampal mitogen-activated protein kinase (MAPK) phosphatase-1 (MKP-1), a negative regulator for ERK activity, caused depressive-like behaviors in rodents with reduced ERK activation [24]. The inactivation of ERK in the dentate gyrus was also observed in depressive-like mice following chronic treatment with corticosterone, and an antidepressant (amitriptyline) restored abnormalities in both ERK signal and behaviors [25]. In concert, activity and expression of ERK were attenuated in the tissues from prefrontal cortex and hippocampus in depressed suicide subjects [26]. Moreover, B-Raf, a Raf kinase family of serine/threonine kinase which regulates ERK signaling, was diminished in the prefrontal cortical and hippocampal tissues from suicide subjects [27]. Additionally, decreased plasma levels of miR-134 in patients with bipolar disorder have also been reported [28]. It is possible that miR-134, which is regulated by ERK signaling, is a useful biomarker for mental disorders, 
though more detailed relationship between ERK activity and miR-134 expression should be clarified. On the other hand, it is well known that the PI3K/Akt signal cascade works for survival promoting in a variety of cell populations [29, 30]. As induction of glial miR-134 by bFGF was also inhibited in the presence of a PI3K inhibitor, cell protection effect might be achieved by miR-134 induction when the astrocyte cultures are exposed to severer conditions including oxidative insult.

It has been demonstrated that elevated hippocampal mRNA expression of FGF receptor 1 was observed in patients with major depressive disorder who exhibited reduced bFGF mRNA expression [31]. Post-mortem studies suggest that a dysfunction of FGF system in the prefrontal cortex is associated with depression [31, 32]. In kainic acid-induced seizure model mice, hippocampal injection of locked nucleic acid (LNA) 3' cholesterol-conjugated oligonucleotides (antagomirs) targeting miR-134 prevents status epilepticus and cell death induction [4]. Indeed, neuronal miRs have a critical role in physiological cellular states including neuronal development and function $[1,2]$. On the other hand, although the astroglial miRs such as miR-125b and -155 have been identified $[9,10]$, physiological function of astroglial miRs is poorly understood. Therefore, it is intriguing to explore whether altered levels of bFGF and miR-134 in brain tissues from depression patients is neuronal or glial response. 
Acknowledgement

This study was supported by grants from by Takeda Science Foundation (T. N.), and from the Grant-in-Aid for Challenging Exploratory Research (JSPS KAKENHI Grant Number 25640019) (T. N.) and the Grant-in-Aid for Scientific Research (B) (JSPS KAKENHI

24300139) (T. N.) in the Ministry of Education, Culture, Sports, Science, and Technology of Japan. This study was also supported by the Health and Labor Sciences Research Grants (H. K.), Intramural Research Grant for Neurological and Psychiatric Disorders of NCNP (H.K.) and CREST, Japan Science and Technology Agency (JST) (T.N., N.A. and H.K.). 


\section{References}

[1] Sun AX, Crabtree GR, Yoo AS. MicroRNAs: regulators of neuronal fate. Curr Opin Cell Biol. 25 (2013) 215-221.

[2] McNeill E, Van Vactor D. MicroRNAs shape the neuronal landscape. Neuron. 75 (2012) 363-379.

[3] Miller BH, Zeier Z, Xi L, Lanz TA, et al. MicroRNA-132 dysregulation in schizophrenia has implications for both neurodevelopment and adult brain function. Proc Natl Acad Sci U S A. 109 (2012) 3125-3130.

[4] Jimenez-Mateos EM, Engel T, Merino-Serrais P, et al. Silencing microRNA-134 produces neuroprotective and prolonged seizure-suppressive effects. Nat Med. 18 (2012) 1087-1094.

[5] Remenyi J, Hunter CJ, Cole C, et al. Regulation of the miR-212/132 locus by MSK1 and CREB in response to neurotrophins. Biochem J. 428 (2010) 281-291.

[6] Numakawa T, Richards M, Adachi N, Kishi S, Kunugi H, Hashido K. MicroRNA function and neurotrophin BDNF. Neurochem Int. 59 (2011) 551-558.

[7] Li L, Chen H, Chen F, Li F, et al. Effects of glial cell line-derived neurotrophic factor on microRNA expression in a 6-hydroxydopamine-injured dopaminergic cell line. J Neural Transm. 120 (2013) 1511-23.

[8] Numakawa T, Yamamoto N, Chiba S, et al. Growth factors stimulate expression of neuronal and glial miR-132. Neurosci Lett. 505 (2011) 242-247.

[9] Pogue AI, Cui JG, Li YY, et al. Micro RNA-125b (miRNA-125b) function in astrogliosis and glial cell proliferation. Neurosci Lett. 476 (2010) 18-22.

[10] Tarassishin L, Loudig O, Bauman A, et al. Interferon regulatory factor 3 inhibits astrocyte inflammatory gene expression through suppression of the proinflammatory miR-155 and miR-155*. Glia. 59 (2011) 1911-1922.

[11] Turner CA, Watson SJ, Akil H. The fibroblast growth factor family: neuromodulation of affective behavior. Neuron. 76 (2012) 160-174. 
[12] Schratt GM, Tuebing F, Nigh EA, et al. A brain-specific microRNA regulates dendritic spine development. Nature. 439 (2006) 283-289.

[13] Gaughwin P, Ciesla M, Yang H, et al. Stage-specific modulation of cortical neuronal development by Mmu-miR-134. Cereb Cortex. 21 (2011) 1857-1869.

[14] Fiore R, Khudayberdiev S, Christensen M, et al. Mef2-mediated transcription of the miR379-410 cluster regulates activity-dependent dendritogenesis by fine-tuning Pumilio2 protein levels. EMBO J. 28 (2009) 697-710.

[15] Numakawa T, Kumamaru E, Adachi N, et al. Glucocorticoid receptor interaction with TrkB promotes BDNF-triggered PLC-gamma signaling for glutamate release via a glutamate transporter. Proc Natl Acad Sci U S A. 106 (2009) 647-652.

[16] Reilly JF, Maher PA, Kumari VG. Regulation of astrocyte GFAP expression by TGF-beta1 and FGF-2. Glia. 22 (1998) 202-210.

[17] Heffron DS, Mandell JW. Opposing roles of ERK and p38 MAP kinases in FGF2-induced astroglial process extension. Mol Cell Neurosci. 28 (2005) 779-90.

[18] Joy A, Moffett J, Neary K, et al. Nuclear accumulation of FGF-2 is associated with proliferation of human astrocytes and glioma cells. Oncogene. 14 (1997) 171-183.

[19] Irmady K, Zechel S, Unsicker K. Fibroblast growth factor 2 regulates astrocyte differentiation in a region-specific manner in the hindbrain. Glia. 59 (2011) 708-719.

[20] Eulenburg V, Gomeza J. Neurotransmitter transporters expressed in glial cells as regulators of synapse function. Brain Res Rev. 63 (2010) 103-112.

[21] Reuss B, Dono R, Unsicker K. Functions of fibroblast growth factor (FGF)-2 and FGF-5 in astroglial differentiation and blood-brain barrier permeability: evidence from mouse mutants. J Neurosci. 23 (2003) 6404-6412.

[22] Sweatt JD. Mitogen-activated protein kinases in synaptic plasticity and memory. Curr Opin Neurobiol. 14 (2004) 311-317. 
[23] Thomas GM, Huganir RL. MAPK cascade signalling and synaptic plasticity. Nat Rev Neurosci. 5 (2004) 173-183.

[24] Duric V, Banasr M, Licznerski P, et al. A negative regulator of MAP kinase causes depressive behavior. Nat Med. 16 (2010) 1328-1332.

[25] Gourley SL, Wu FJ, Kiraly DD, et al. Regionally specific regulation of ERK MAP kinase in a model of antidepressant-sensitive chronic depression. Biol Psychiatry. 63 (2008) 353-359.

[26] Dwivedi Y, Rizavi HS, Roberts RC, et al. Reduced activation and expression of ERK1/2 MAP kinase in the post-mortem brain of depressed suicide subjects. J Neurochem. 77 (2001) 916-928.

[27] Dwivedi Y, Rizavi HS, Conley RR, et al. ERK MAP kinase signaling in post-mortem brain of suicide subjects: differential regulation of upstream Raf kinases Raf-1 and B-Raf. Mol Psychiatry. 11 (2006) 86-98.

[28] Rong H, Liu TB, Yang KJ, et al. MicroRNA-134 plasma levels before and after treatment for bipolar mania. J Psychiatr Res. 45 (2011) 92-95.

[29] Amaravadi R, Thompson CB. The survival kinases Akt and Pim as potential pharmacological targets. J Clin Invest. 115 (2005) 2618-2624.

[30] Frebel K, Wiese S. Signalling molecules essential for neuronal survival and differentiation. Biochem Soc Trans. 34 (2006) 1287-1290.

[31] Gaughran F, Payne J, Sedgwick PM, et al. Hippocampal FGF-2 and FGFR1 mRNA expression in major depression, schizophrenia and bipolar disorder. Brain Res Bull. 70 (2006) 221-227.

[32] Tochigi M, Iwamoto K, Bundo M, et al. Gene expression profiling of major depression and suicide in the prefrontal cortex of postmortem brains. Neurosci Res. 60 (2008) 184-191. 


\section{Figure Legends}

Figure $1 \mathrm{bFGF}$ induced upregulation of miR-134 in astrocytes.

(A) The expression of miRs (miR-132, -9, -124a, and -134) in cortical neurons after BDNF or bFGF stimulation. Cortical neurons at DIV 4-5 were exposed to $100 \mathrm{ng} / \mathrm{ml}$ BDNF or bFGF for $24 \mathrm{~h}(\mathrm{n}=4)$, respectively. (B) Changed expression of miRs in astroglia after bFGF incubation for $24 \mathrm{~h}$ at $100 \mathrm{ng} / \mathrm{ml}(\mathrm{n}=5)$. (C) Dose-dependency of astroglial miR-134 upregulation. bFGF (1-100 ng/ml) was added (n=6). (D) BDNF, bFGF, IGF-1, GDNF, or EGF were applied to astroglial cultures to evaluate their effect on miR-134 induction $(n=6)$. The expressions of miRs were determined with real-time PCR. Error bars represent SD. ${ }^{*} P<0.05,{ }^{* *} P<0.01,{ }^{* * *} P<$ 0.001 .

Figure 2 The involvement of MAPK/ERK and PI3K signaling cascades in the bFGF-induced miR-134.

(A) Activated ERK1/2 (phosphorylated ERK1/2, pERK1/2) and total ERK1/2 by bFGF were examined. Astrocytes were exposed to bFGF $(100 \mathrm{ng} / \mathrm{ml}$, for $20 \mathrm{~min})$ in the presence or absence of U0126 $(10 \mu \mathrm{M})$. (B) Astroglial miR-134 expression after treatment with $100 \mathrm{ng} / \mathrm{ml} \mathrm{bFGF}$ in the presence or absence of U0126 (n=4-6). (C) Activated Akt (phosphorylated Akt) and total Akt after bFGF application. Astrocytes were exposed to $100 \mathrm{ng} / \mathrm{ml} \mathrm{bFGF}$ for $20 \mathrm{~min}$ with or without LY294002 (10 $\mu$ M). (D) Astroglial miR-134 levels after bFGF stimulation with or without LY294002 (n=4). Error bars represent SD. ${ }^{* * *} P<0.001$ vs control, ${ }^{\dagger \dagger} P<0.01,{ }^{\# \#} P<0.001$.

Figure 3 The effect of miR-134 overexpression on astroglial cell survival.

(A) The levels of miR-134 after control- or miR-134-coding vectors were transfected into astrocytes $(\mathrm{n}=5)$. (B) Cell survival after control- or miR-134-coding vectors transfection. Error bars represent SD. (N=8). Cell viability was measured with MTT assay. (C) The activated levels of ERK and Akt signaling in control- or miR-134-coding vector transfected astrocytes $(n=5)$. The values are mean $\pm \mathrm{SD}$. ${ }^{* * *} P<0.001$ vs control.

Figure 4 Overexpression of miR-134 enhanced expression of glial markers and glutamate transporter, and decreased extracellular glutamate concentration.

(A) Immunostaining with anti-GFAP antibody in control- or in miR-134 expression vector-transfected astrocytes. The immunofluorescence intensity was measured using control$(n=32)$ or miR-134-overexpressed cells $(n=37)$ from 4 dishes for each condition. (B) Glial marker proteins (GFAP, ALDH1L1, and S-100) expression in control or miR-134 overexpression in astrocytes $(n=5)$. (C) The expression levels of GFAP and ALDH1L1 at 24 hours after bFGF (100 ng/ml) application in astrocytes (n=5). (D) miR-134 overexpression and 
(E) bFGF application increased GLT-1 expression in astrocyte ( $n=5)$. (F) Reduced concentration of extracellular glutamate in astroglial cultures after miR-134 overexpression and bFGF application $(\mathrm{n}=6-12)$. The values are mean $\pm \mathrm{SD} .{ }^{* *} \mathrm{P}<0.01,{ }^{* * *} \mathrm{P}<0.001$ vs control. 
Figure 1

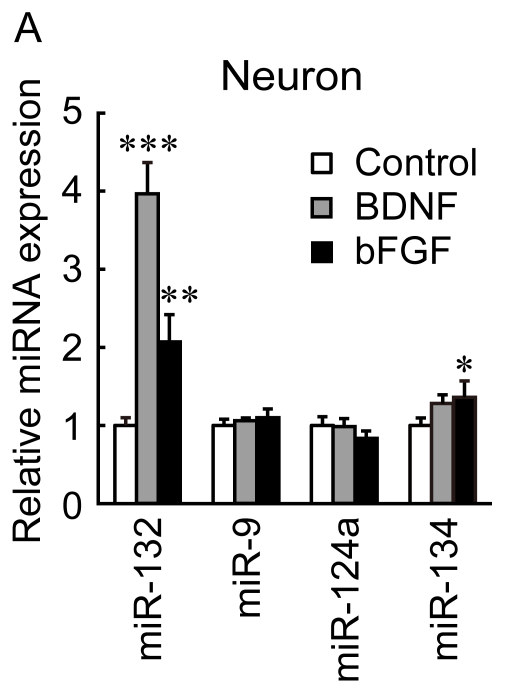

B

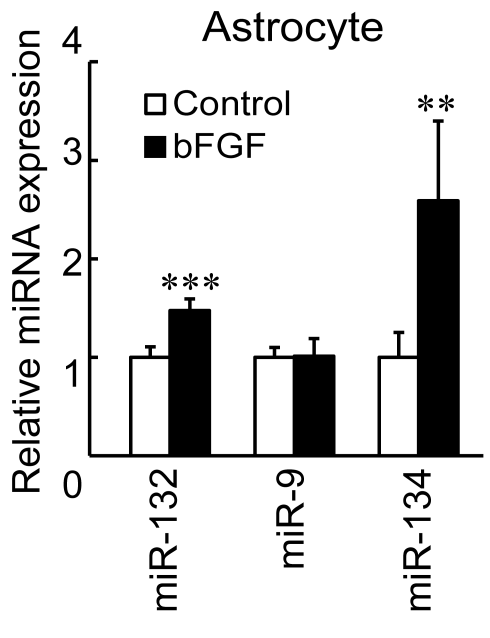

C

D

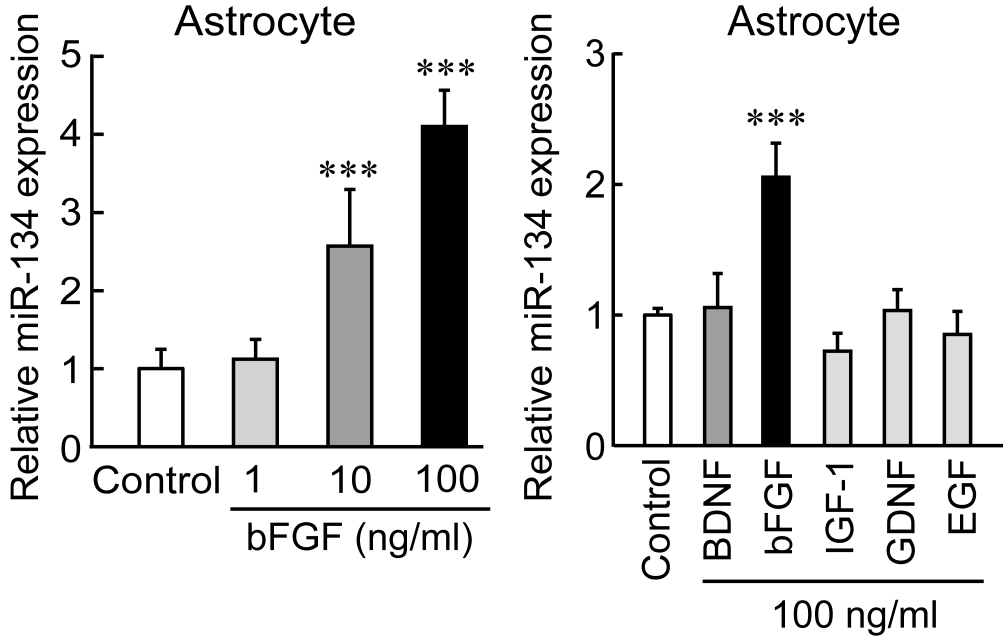


A

B

pERK1 -

pERK2 -

ERK1 -

ERK2

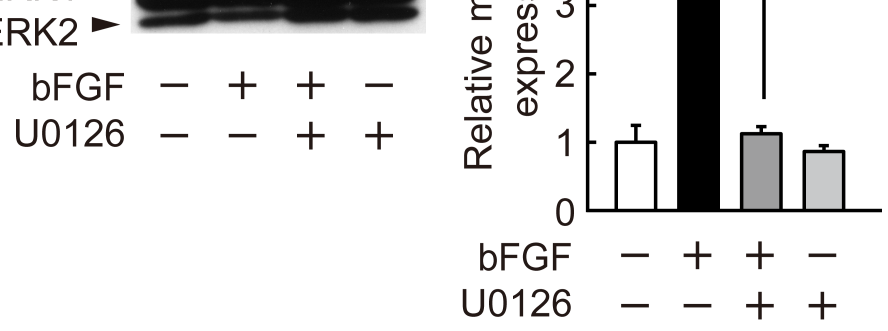

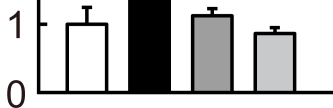

C

D

pAkt -

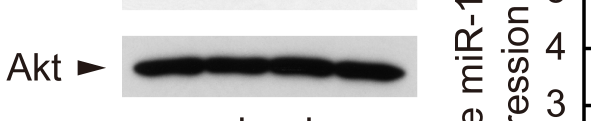

bFGF $-++-\sum_{\geq}^{0} \frac{0}{x} 2$

LY294002 - + + $\underset{\mathbb{Q}}{\mathbb{N}}$

bFGF -++-

LY294002 - - + + 
A

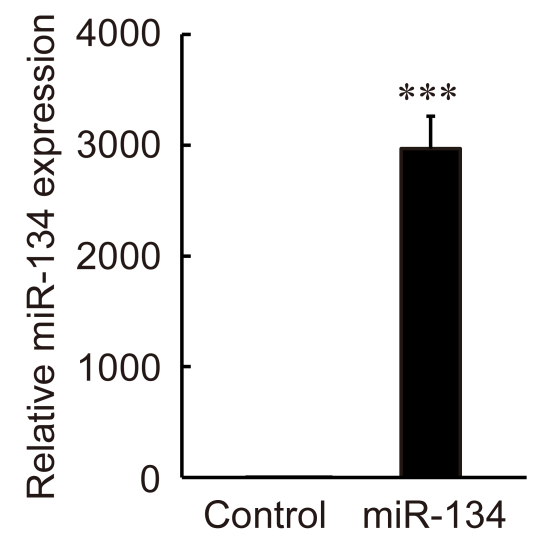

B

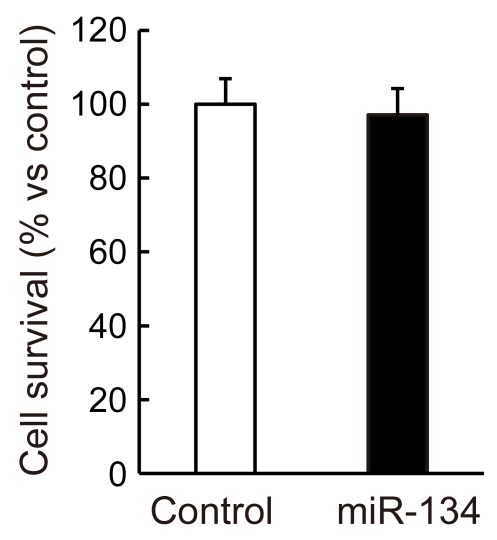

C
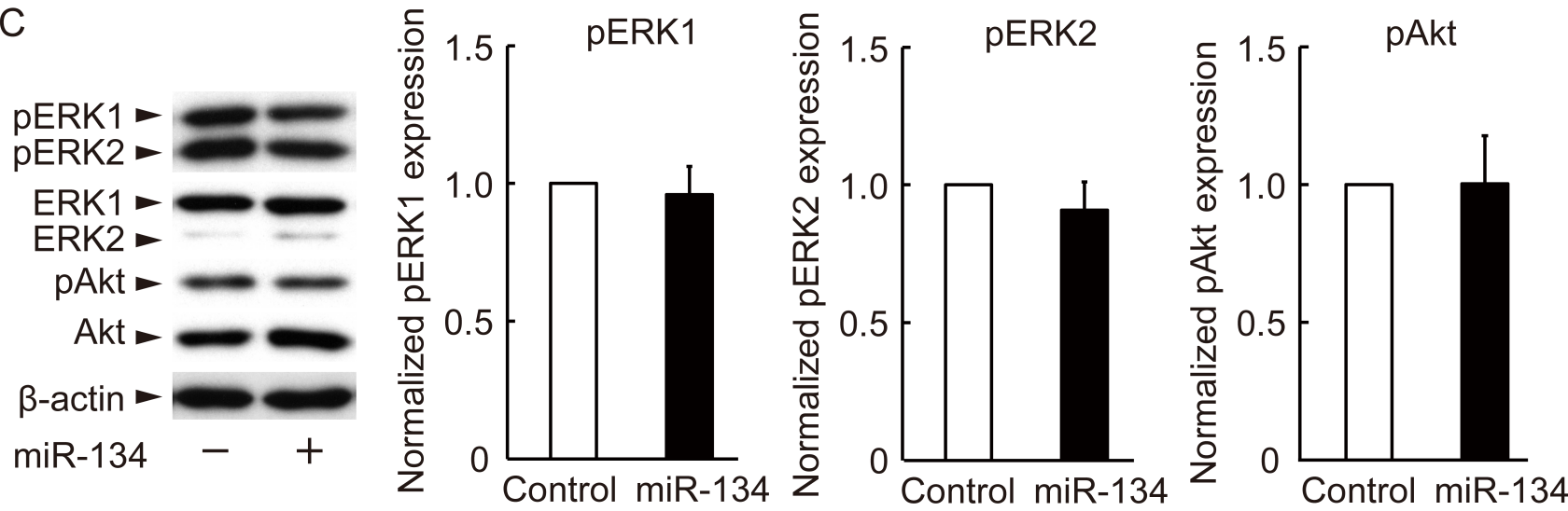


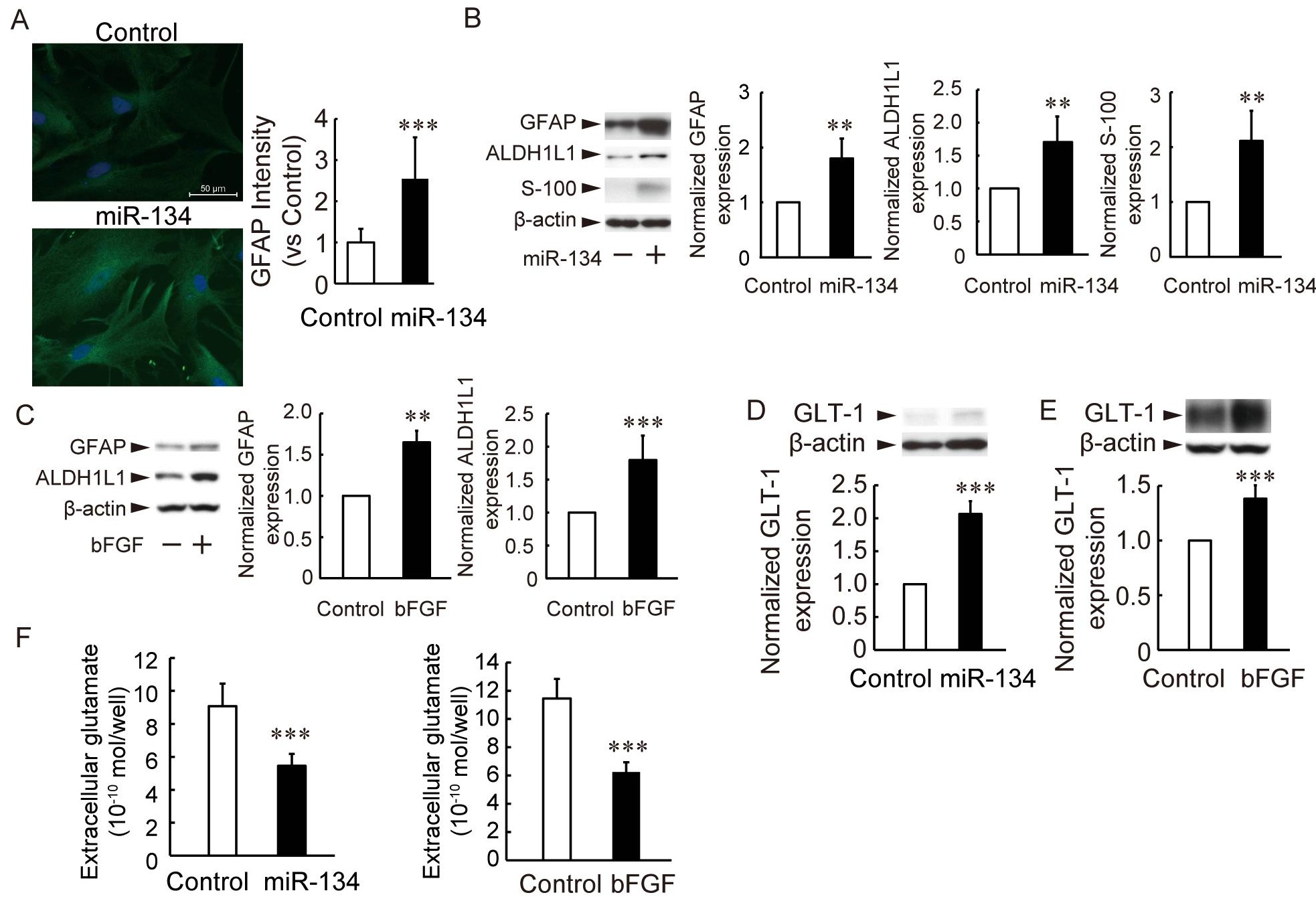

\section{A}

Control miR-134
GLT-1 - 